\title{
Functional Leukocyte Administration in Protection against Experimental Neonatal Infection
}

\author{
JOSÉ IGNACIO SANTOS, ${ }^{(22)}$ ANN O. SHIGEOKA, AND HARRY R. HILL \\ Howard Hughes Medical Institute Laboratory, Division of Clinical Immunology and Allergy, Department of \\ Pediatrics, and the Department of Pathology, University of Utah College of Medicine, Salt Lake City, Utah, USA
}

\begin{abstract}
Summary
Studies have shown that human neonates who develop group B streptococcal sepsis usually lack opsonic antibody (Ab) to their infecting strain and that these neonates may also have impaired polymorphonuclear leukocyte (PMN) function. The present study was designed to determine the efficacy of administration of PMNs or opsonic Ab-containing serum in protecting against group B streptococcal infection in a newborn rat model. After intraperitoneal (IP) injection of $\simeq 5 \times 10^{6}$ streptococci, animals received separate IP injections of saline, serum lacking opsonic antibody (Ab negative), Ab positive serum or washed adult human PMNs $\left(2 \times 10^{6}\right)$. The mortality rate in 55 neonatal rats infected with group B streptococci who received saline or Ab negative serum was 91\%. In contrast, 40 animals who received adult human PMNs at the time of inoculation had a survival rate of $50 \%(P<0.001)$. Human serum containing opsonic antibody also provided significant protection against mortality in this model (survival rate 51\%, $P<0.001$ ). Leulocytes from normal term neonates, stressed neonates, or ones pretreated with cytochalasin B offered less protection than did functional adult human cell $(P<0.001)$.
\end{abstract}

\section{Speculation}

This study provides evidence for polymorphonuclear leukocyte (PMN) dysfunction as another risk factor in group B streptococcal disease and suggests that fully functional PMNs are required for protection. Furthermore, this model involving the administration of human PMNs to an animal appears to have the potential for the in vivo evaluation of PMN function in neonates and other compromised hosts.

The mechanisms of host resistance to streptococci of Lancefield's group B have not been precisely defined. Mouse protection studies by Lancefield et al. $(14,15)$ and Fischer $e t$ al. (9) have shown the protective effect of antibodies against group B streptococci. Hemming et al. (10), Shigeoka et al. (17), and Anthony (2) have demonstrated the absolute requirement of specific antibody for significant opsonization of group B streptococci. In previous studies, we have shown that human neonates who develop group B streptococcal sepsis usually lack opsonic antibody to their infecting strains (10) and that these neonates may also have impaired polymorphonuclear leukocyte function (19). Inasmuch as opsonization and phagocytosis represent the most important host defense mechanisms in clearing the body of these bacteria, the present study was designed to determine the effects of passively supplying functional human polymorphonuclear leukocytes (PMNs) or serum containing opsonic antibody in protecting against group B streptococcal infection in a newborn rat model.

\section{METHODS}

PREPARATION OF ORGANISMS

Group B streptococci type III were isolated from the blood and cerebrospinal fluid of infected infants. The strains were cultured at $37^{\circ} \mathrm{C}$ in Todd-Hewitt broth (Difco Laboratories, Detroit, $\mathrm{MI}$ ) washed and concentrated in phosphate-buffered saline by centrifugation to contain $5.0 \times 10^{8}$ to $1.0 \times 10^{9}$ colony-forming units per $\mathrm{ml}$, as previously described $(10,17)$.

\section{NEONATAL RAT INFECTIONS}

Sprague-Dawley outbred pregnant female rats (Holtzman) were obtained one wk before delivery. When tested, maternal rat serum did not appear to contain significant opsonic activity against most of the strains we used. The neonatal progeny less than $24 \mathrm{hr}$-old were randomly assigned to groups and entered into the study. After intraperitoneal (IP) injection of $5.0 \times 10^{6}$ streptococci with a Hamilton syringe, the animals received separate IP equal volume $(10 \mu \mathrm{l})$ injections of saline, Ab negative serum, Ab positive serum, washed adult human PMNs $\left(2 \times 10^{6}\right)$ or PMNs pretreated with cytochalasin $B$ and washed thoroughly. The rat litters were observed at 6-hr intervals for mortality. Those neonatal rats who died were aseptically dissected, and in all instances in which cultures were taken, group B streptococci were cultured from one or several of the following organs: brain, lungs, kidney, liver, and spleen.

\section{PREPARATION OF PMN}

Heparinized blood ( 10 units $/ \mathrm{ml}$ ) was collected from healthy adult volunteers, gravity sedimented to remove erythrocytes, and washed twice with PBS. PMNs were then counted and adjusted to a concentration of $1.0 \times 10^{7} / \mathrm{cc}$ representing 50 to $70 \%$ of the cells in the suspension. In a similar fashion, leukocytes were collected and prepared from cord blood of noninfected term human neonates and from neonates whose delivery was stressed by maternal preeclampsia or premature rupture of membranes.

\section{ASSESSMENT OF NEUTROPHIL VIABILITY AFTER IP INJECTION}

Latex particles, $0.81 \mu$ (Difco Laboratories) were washed once in $10 \mathrm{ml}$ PBS, and the pelllet was resuspended in PBS to a concentration of $1 \times 10^{4}$ in a sterile plastic tube. Group B streptococci type III were prepared as previously described. Sprague-Dawley outbred adult and neonatal rats were injected IP with equal volume injections $(10 \mu \mathrm{l})$ of either latex particles or group B streptococci before receiving washed adult human PMNs $\left(2 \times 10^{6}\right)$. Thirty min after injection, animals underwent peritoneal washout with $10 \mathrm{ml}$ (adults) or $2 \mathrm{ml}$ (neonates) sterile PBS. The exudates were cytocentrifuged onto slides and visual evidence of phagocytic uptake of either latex particles or group B streptococci was evaluated by examination of Giemsa-stained smears (10).

\section{SERUM TREATMENT}

Fresh human serum or frozen serum (at $-70^{\circ} \mathrm{C}$ thawed only on use) was utilized. Opsonic antibody titers were determined by a chemiluminescence procedure developed in our laboratory (10) and confirmed in most instances by a radio-labeled bacterial uptake technique (1). 
RESULTS

The survival rate in 55 neonatal rats infected with group B streptococci, type III, who received saline or opsonic antibody negative serum was only $9 \%$ (Table 1 ). In contrast, animals who received human serum containing opsonic antibody to the infecting strain had a significantly improved survival rate $(50 \%)$. Similarly, a marked increase in survival was also observed in animals who received group B streptococci and a separate intraperitoneal injection of $2 \times 10^{6}$ adult human PMNs. Thus, both human serum containing opsonic antibody to the infecting strain as well as functional adult human leukocytes significantly improve survival in infected neonatal rats. Saline and opsonic antibody negative serum did not decrease mortality. All 30 uninfected animals who received human leukocytes alone survived.

Giemsa-stained smears of peritoneal exudates from both adult and neonatal rats revealed that human PMNs (evidenced by relative size and nuclear configuration) had phagocytized both latex particles and group B streptococci equally well. Thus, the human cells remained viable and functionally active within the rat peritoneal cavity.

In addition to investigating adult humoral and cellular factors in the immune response to group B streptococci, we also evaluated PMNs from stressed human neonates because we and others have previously detected metabolic, chemotactic, and bactericidal defects in these cells $(13,16,19)$. The data in Figure 1 is a comparison between the protection provided by adult and neonatal PMNs in newborn rats infected with a different strain of type III group B streptococci. As shown, adult PMNs provided significant protection against the infecting strain resulting in an overall survival rate of $69 \%$ versus $0 \%$ in the saline-treated animals. In contrast, the same number of PMNs from stressed neonates showed much less protection with an overall survival of only $28 \%$. The difference in protection supplied by the functional adult PMNs and the stressed neonatal PMNs is significant at $P<0.001$. In a similar fashion we next compared the protective effect of adult PMNs with the

Table 1. Effect of administering functional leukocytes or opsonic antibody on the survival of group $B$ infected rats

\begin{tabular}{lccc}
\hline \multirow{2}{*}{ Group } & \multicolumn{3}{c}{$\%$ survival at $96 \mathrm{hr}$} \\
\cline { 2 - 4 } \multicolumn{1}{c}{$n$} & & $P^{1}$ \\
\hline GBS $^{2} \pm$ saline or $\mathrm{AB}^{-}$serum & 55 & $5 / 55(9)^{3}$ & \\
${\text { GBS + } \mathrm{AB}^{+} \text {serum }}_{\text {GBS + PMNs }}^{40}$ & $20 / 40(50)$ & $<0.001$ \\
\hline
\end{tabular}

${ }^{1} P=$ Fisher exact test.

${ }^{2} \Delta \mathrm{GBS}$, Group B streptococci.

${ }^{3}$ Numbers in parentheses, percent.

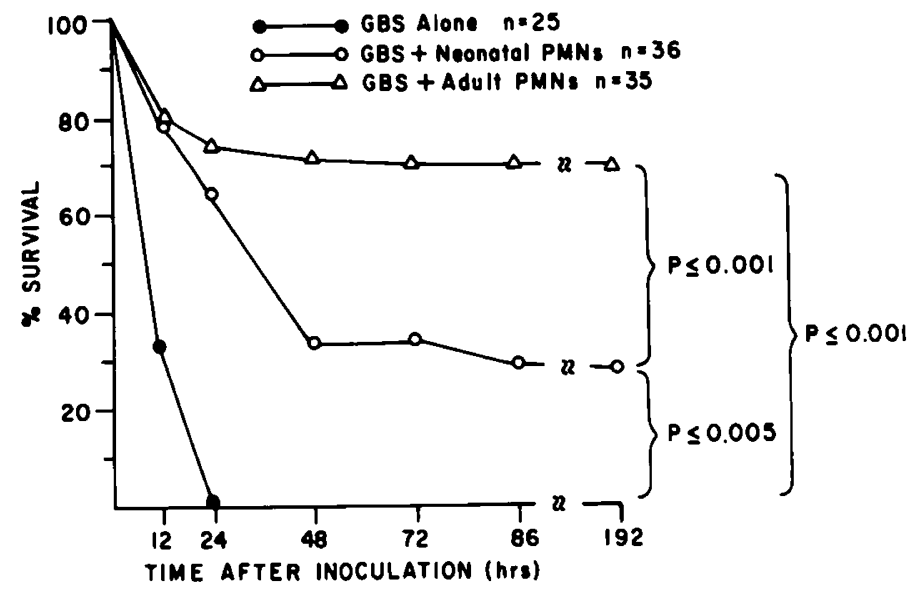

Fig. 1. Effect of leukocyte administration from human stressed neonates and adult controls on survival of neonatal rats infected with type III group B streptococci (GBS).

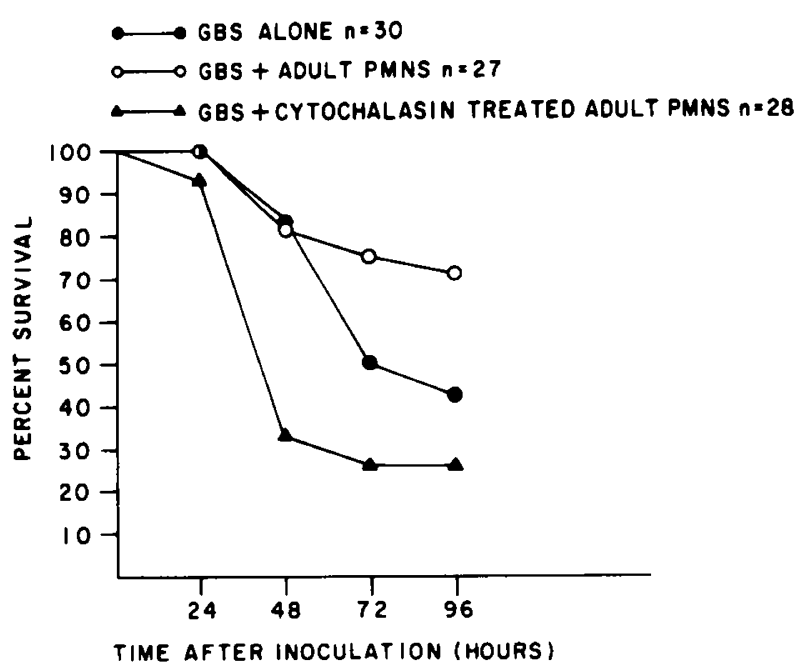

Fig. 2. Effect of cytochalasin treatment of leukocytes on neonatal rat protection in type III group B streptococcus (GBS) infection.

same number of PMNs from uninfected, healthy, term neonates. The survival rate in 28 infected animals who received adult PMNs was $50 \%$ ( 14 of 28 ) compared to $25 \%$ ( 8 of 32 ) in animals receiving PMNs from healthy, term neonates.

To further confirm that the administered leukocytes must be functional to improve survival in the neonatal rat model, adult human leukocytes were incubated with cytochalasin B (Aldrich Chemical Co., Milwaukee, WI) in concentrations $(10 \mu \mathrm{g} / \mathrm{ml})$ known to have an effect on both chemotaxis and phagocytosis in vitro $(5,7)$. The protective effect of these cells was compared with that of untreated leukocytes. The survival rate in 27 neonatal rats infected with group B streptococci who received adult human PMNs pretreated with cytochalasin B was only $26 \%$, whereas animals receiving untreated PMNs had a survival rate of $71 \%$ (Fig. 2; $P<0.001$ ). Pretreatment of leukocytes with cytochalasin appears, therefore, to inhibit PMN antibacterial function when tested in vivo in this animal model.

\section{DISCUSSION}

Group B streptococci have emerged over the last decade as a major cause of morbidity and mortality in neonates. Group B disease is known to occur in other compromised hosts including diabetics $(4,8)$, patients with liver disease, and ones with renal failure (4). Moreover, we have demonstrated that acutely stressed neonates with group B streptococcal sepsis or meningitis often develop neutropenia (6) and have PMN chemotactic and bactericidal abnormalities (19). Clinical studies relating neonatal and maternal antibody levels to infection have previously suggested the importance of antibody in host defense against these strains $(3,10)$. Similarly, limited observations $(18)$ have shown that infants infected with group B streptococci who received transfusions of whole blood containing opsonic antibody to the infecting strain had improved survival when compared to infants receiving blood lacking antibody to their infecting organism.

Studies in animals have also suggested the importance of antibody in the protection against group B disease $(9,14,15)$. Our data indicate that human PMNs are both viable and capable of phagocytosis after being introduced into the peritoneal cavity of both adult and neonatal rats. For these reasons, we have utilized a neonatal rat model of group B disease and have sought to determine the effects of opsonic antibody and leukocyte administration on the survival of these experimentally infected animals.

Our data indicate that functional human leukocyte administration affords protection in this animal model. Caution should be exercised, however, in extrapolating the clinical applicability of our results to the human neonate. We are not recommending leukocyte transfusion in such patients. It is conceivable, however, that for limited conditions such as overwhelming group B streptococcal sepsis the combination of opsonic antibody and func- 
tional cellular elements (PMNs) may prove to be efficacious. Considerable additional work in animal models such as the one described here needs to be carried out before clinical trials can even be considered. Then the problem of possible graft versus host disease or transmission of infection via blood and blood products will have to be considered. Still, such avenues should be investigated in attempts to improve survival in this type of overwhelming infection with mortality rates in the 25 to $75 \%$ range. The study also provides evidence for PMN dysfunction being another risk factor in group B disease because neonatal animals given antibody to their infecting strain often still had a mortality rate of $\mathbf{4 0}$ to $50 \%$. We were also able to show that human neonatal PMNs were less effective in providing protection in this animal model.

PMNs from healthy term neonates behave very similar to those from normal adult controls in assays of chemiluminescence generation or bactericidal activity when tested in vitro (19). In contrast, PMNs from stressed neonates have a subtle bactericidal defect when challenged with a high bacteria to PMN ratio. The present observations suggest that PMNs from both healthy term and stressed neonates are deficient in their ability to protect newborn rats from group B streptococcal infection. Giemsa-stained smears of peritoneal exudates from neonatal rats revealed that human PMNs from adult controls as well as healthy term and stressed neonates are capable of phagocytizing both latex particles and group B streptococci equally well. Inasmuch as healthy and stressed neonatal PMNs are both deficient in their ability to protect neonatal rats, it is unlikely that the subtle bactericidal defect observed in vitro in stressed PMNs contributes to this phenomenon. Both healthy and term neonatal PMNs have defective chemotactic activity $(13,16)$ which would partially explain the abnormality observed. Of interest is the fact that diabetic individuals who have an increased incidence of group B streptococcal infections $(4,8)$ also have defects in PMN chemotactic activity $(11,12)$.

Additional studies with cytochalasin B tended to support the conclusions that functional PMNs are required for protection in this model. Cytochalasin B has been reported to inhibit spontaneous motility and chemotactic responses of rabbit and human leukocytes (5). Davis et al. (7) also reported the inhibition of bacterial phagocytosis by human polymorphonuclear leukocytes in the presence of cytochalasin B. Our results support these observations and aid in delineating the importance of PMN chemotaxis and phagocytosis in the host defense mechanism against group B streptococci. The data from these experiments also confirm that significant protection in this model results only when functional PMNs are utilized. Furthermore, this model involving the administration of human PMNs to an animal appears to have the potential for the in vivo evaluation of PMNs from neonates and other compromised hosts.

\section{REFERENCES AND NOTES}

1. Allred, C. D., Shigeoka, A. O., and Hill, H. R.: Evaluation of group B streptococcal opsonins by radiolabeled bacterial uptake. J. Immunol. Methods, 26: 355 (1979).

2. Anthony, B. F.: Immunity to the group B streptococci: Interaction of serum and macrophages with types Ia, Ib, and Ic. J. Exp. Med., 143: 1186 (1976).

3. Baker, C. J., and Kasper, D. L.: Correlation of maternal antibody deficiency with susceptibility to neonatal group B streptococcal infection. N. Engl. J. Med., 294: 753 (1976).

4. Bayer, A. S., Chow, A. W., Anthony, B. F., and Guze, L. B.: Serious infections in adults due to group B streptococci. Am. J. Med., 61: 498 (1976).

5. Becker, E. L., Davis, A. T., Estensen, R. D., and Quic, P. G.: Cytochalasin B. IV Inhibition and stimulation of chemotaxis of rabbit and human polymorphonuclear leukocytes. J. Immunol., I08: 396 (1972).

6. Christensen, R. D., Shigeoka, A. O., Hill, H. R., and Rothstein, G.: Neutropenia and bone marrow exhaustion in human and experimental neonatal sepsis. Pediatr. Res., 14: 806 (1980)

7. Davis, A. T., Estensen, R., and Quie, P. G.: Cytochalasin B. III. Inhibition of human polymorphonuclear leukocyte phagocytosis. Proc. Soc. Exp. Biol. Med. 137: 161 (1971).

8. Eickhoff, T. C., Klein, J. O., Daly, A. K., Ingall, D., and Finland, M.: Neonatal sepsis and other infections due to group B beta-hemolytic streptococci. N. Engl. J. Med., 271: 1221 (1964).

9. Fischer, G. W., Lowell, G. H., Crumrine, M. H., and Bass, J. W.: Demonstration of opsonic activity and in vivo protection against group B streptococci type II by Streptococcus pneumoniae type 14 antisera. J. Exp. Med. 148: 776 (1978).

10. Hemming, V. G., Hall, R. T., Rhodes, P. G., Shigeoka, A. O. and Hill, H. R. Assessment of group B streptococcal opsonins in human and rabbit serum by neutrophil chemiluminescence. J. Clin. Invest., 58 : 1379 (1976).

11. Hill, H. R., Sauls, H. S., Dettloff, J. L., and Quie, P. G.: Impaired leukotactic responsiveness in patients with juvenile diabetes mellitus. Clin. Immunol. Immunopathol., 3: 395 (1974).

12. Hogan, N. A., Rallison, M. L., Santos, J. I., and Hill, H. R.: Abnormal monocyte chemotactic responsiveness in diabetes mellitus. Clin. Res., 28: 108A (1980).

13. Klein, R. B., Fischer, T. J., Gard, S. E., Biberstein, M., Rich, K. C., and Stiehm, E. R.: Decreased mononuclear and polymorphonuclear chemotaxis in human newborns, infants, and young children. Pediatrics, 60: 467 (1977).

14. Lancefield, R. C., and Freimer, E. H.: Type specific polysaccharide antigens of group B streptococci. J. Hyg. (Cambridge), 64: 191 (1966).

15. Lancefield, R. C., McCarty, M., and Everly, W. N.: Multiple mouse-protective antibodies directed against group B streptococci. J. Exp. Med., 142: 165 (1975).

16. Miller, M. E.: Chemotactic function in the human neonate. Humoral and cellular aspects. Pediatr. Res., 5: 487 (1971).

17. Shigeoka, A. O., Hall, R. T., Hemming, V. G., Allred, C. D., and Hill, H. R.: Role of antibody and complement in opsonization of group B streptococci. Infect. Immun., $21: 34$ (1978)

18. Shigeoka, A. O., Hall, R. T., and Hill, H. R.: Blood-transfusion in group B streptococcal sepsis. Lancet, 1: 636 (1978).

19. Shigeoka, A. O., Santos, J. I., and Hill. H. R.: Functional analysis of neutrophil granulocytes from healthy, infected and stressed neonates. J. Pediatr., 95: 454 (1979).

20. Presented, in part, at the meeting of the Society for Pediatric Research, Atlanta, May 4, 1979.

21. The authors thank Val Callanan for secretarial assistance.

22. Requests for reprints should be addressed to: José Ignacio Santos, M.D., Maxwell Finland Laboratory for Infectious Diseases, 774 Albany St., Boston City Hospital, Boston, MA, 02118 (USA)

23. This research was supported in part by United States Public Health Service Grants AI13150 and AM21140 and a grant from the Thrasher Foundation.

24. Received for publication February 1, 1980

25. Accepted for publication May 19, 1980. 